\title{
Optical characterization of metallic aerosols
}

\author{
Wenbo Sun \\ Center for Atmospheric Sciences, Hampton University, Hampton, Virginia 23668 \\ Bing Lin \\ Atmospheric Sciences Research, NASA Langley Research Center, Hampton, Virginia 23681
}

Submitted to J. Quant. Spectrosc. Radiat. Transfer

October 1, 2005

Corresponding author address: Dr. Wenbo Sun, Mail Stop 420, NASA Langley Research Center, Hampton, VA 23681-0001. E-mail: w.sun@ larc.nasa.gov 


\begin{abstract}
Airborne metallic particulates from industry and urban sources are highly conducting aerosols. The characterization of these pollutant particles is important for environment monitoring and protection. Because these metallic particulates are highly reflective, their effect on local weather or regional radiation budget may also need to be studied. In this work, light scattering characteristics of these metallic aerosols are studied using exact solutions on perfectly conducting spherical and cylindrical particles. It is found that for perfectly conducting spheres and cylinders, when scattering angle is larger than $\sim 90^{\circ}$ the linear polarization degree of the scattered light is very close to zero. This light scattering characteristics of perfectly conducting particles is significantly different from that of other aerosols. When these perfectly conducting particles are immersed in an absorbing medium, this light scattering characteristics does not show significant change. Therefore, measuring the linear polarization of scattered lights at backward scattering angles can detect and distinguish metallic particulates from other aerosols. This result provides a great potential of metallic aerosol detection and monitoring for environmental protection.
\end{abstract}

Key words: Light scattering, metallic aerosol, environmental protection. 


\section{Introduction}

Metallic aerosols are frequently found in urban or industrialized regions. The concentrations of this type of aerosols are closely associated with weather conditions [1]. Airborne metallic particulates are highly conducting aerosols and can be especially harmful. They may introduce serious human diseases. They can also cause permanent or intermittent failures on electronic equipments. This problem is exaggerated by the increasingly dense circuitry of modern electronic equipment. The characterization of these pollutant particles is important for environment monitoring and protection. Because these metallic particulates are highly reflective, their effect on local weather or regional radiation budget may also need to be studied. In this work, light scattering characteristics of metallic aerosols are studied using exact solutions on perfectly conducting spherical and cylindrical particles. In Section 2, we will present the algorithms to calculate light scattering phase matrix elements of perfectly conducting spheres and cylinders. In Section 3, the light scattering phase matrix elements of perfectly conducting spheres and cylinders are compared with those of sand dusts. Conclusions are given in Section 4.

\section{Methodology}

Because of their extreme conductivity, metallic aerosols can be approximated by perfectly conducting particles, and the light scattering characteristics of perfectly conducting particles can be used to characterize metallic aerosols. The elements of Mueller matrix, which are measurable in field experiments, are calculated from the amplitude scattering matrix [2]. The formulation of amplitude scattering matrix for perfectly conducting spheres [3,4] and circular cylinders [5, 6] are presented in this 
section. For general nonspherical metallic aerosols, due to their extreme conductivity, there are no accurate analytical or numerical solutions available to date. For example, the discrete-dipole approximation (DDA) method [7-9] simply cannot be applied for perfectly conducting particles, because of the zero inside fields. The finite-difference time-domain (FDTD) technique [10-13] can be applied to calculate the near fields scattered by the metallic particles; but also due to the zero inside fields, the volume integral transformation of near fields to far fields cannot be applied, whereas the virtualsurface integral transformation involves significant errors because of the FDTD errors in the forward-scattering near fields [14]. Therefore, an accurate calculation of light scattering by general scattering systems composed of nonspherical particles and involving multiple scattering will still be a challenging problem in the foreseeable future.

\section{A. Spheres}

For light scattering by a perfectly conducting spherical particle with a radius of $a$ embedded in a dielectric medium with a complex refractive index of $m$, the scattering coefficients $a_{n}$ and $b_{n}$ can be derived by assuming zero fields inside the particle. Following Fu and Sun [15], we have

$$
\begin{gathered}
a_{n}=\frac{\psi_{n}^{\prime}(m x)}{\xi_{n}^{\prime}(m x)}, \\
b_{n}=\frac{\psi_{n}(m x)}{\xi_{n}(m x)},
\end{gathered}
$$

where $x=2 \pi a / \lambda_{0}$ and $\lambda_{0}$ denotes the incident wavelength in free space; the RiccatiBessel functions $\psi_{n}(\rho)=\rho j_{n}(\rho)$ and $\xi_{n}(\rho)=\rho h_{n}^{(1)}(\rho)$, where $j_{n}$ and $h_{n}^{(1)}$ denote the 
spherical Bessel function of the first kind and the spherical Hankel function, respectively.

For a spherical particle, the nonzero elements of the amplitude scattering matrix are derived using a far-field approximation as $[2,15]$

$$
\begin{aligned}
& s_{1}(\theta)=\sum_{n=1}^{\infty} \frac{2 n+1}{n(n+1)}\left[a_{n} \pi_{n}(\cos \theta)+b_{n} \tau_{n}(\cos \theta)\right], \\
& s_{2}(\theta)=\sum_{n=1}^{\infty} \frac{2 n+1}{n(n+1)}\left[a_{n} \tau_{n}(\cos \theta)+b_{n} \pi_{n}(\cos \theta)\right],
\end{aligned}
$$

where $\theta$ is the scattering angle; $\pi_{n}=P_{n}^{1} / \sin \theta$ and $\tau_{n}=d P_{n}^{1} / d \theta$ and $P_{n}^{1}$ is the associated Legendre functions of the first kind of degree $n$ and order one.

\section{B. Circular Cylinders}

Most particles in nature have nonspherical shapes which include cylinder-like particulates in air or water. For an infinite perfectly conducting circular cylinder with a radius of $a$ immersed in a dielectric medium with a complex refractive index of $m$, which is illuminated by a normally-incident plane wave, the scattering coefficients $a_{n}$ and $b_{n}$ for the two orthogonal polarization states of the incident wave are derived following Sun et al. [16] as

$$
\begin{aligned}
& a_{n}=\frac{J_{n}^{\prime}(m x)}{H_{n}^{(1)^{\prime}}(m x)}, \\
& b_{n}=\frac{J_{n}(m x)}{H_{n}^{(1)}(m x)},
\end{aligned}
$$

where $x=2 \pi a / \lambda_{0} ; J_{n}(\rho)$ and $H_{n}^{(1)}(\rho)$ denote Bessel function of the first kind the Hankel function, respectively. 
For a circular cylinder, the nonzero elements of the amplitude scattering matrix can be derived from the far-field approximation as $[2,16]$

$$
\begin{aligned}
& s_{1}(\theta)=a_{0}+2 \sum_{n=1}^{\infty} a_{n} \cos (n \theta), \\
& s_{2}(\theta)=b_{0}+2 \sum_{n=1}^{\infty} b_{n} \cos (n \theta) .
\end{aligned}
$$

In the far-field zone, the normalized phase matrix is from the Mueller matrix normalized by the local scattering rate. The formulations of other single scattering properties of perfectly conducting spheres or circular cylinders, such as extinction or scattering efficiency, single-scattering albedo, and asymmetry factor, are identical to those reported in Fu and Sun [15] or in Sun et al. [16].

\section{Results}

We calculated the elements of the normalized scattering phase matrix for perfectly conducting spheres and circular cylinders in both free space and in an absorbing medium. To demonstrate the characteristics of light scattering by metallic particles, we also show the elements of the normalized scattering phase matrix for sand aerosols. The computational programs developed in Fu and Sun [15] and in Sun et al. [16] are used in the calculation of light scattering properties for these sand spheres and circular aerosols, respectively. In this study, we assume a refractive index of 1.5 for sand. When an absorbing medium is assumed around the particles, the refractive index of the host medium is assumed to be $m=1.33+0.01 \mathrm{i}$. The elements of the normalized scattering 
phase matrix shown here are $\mathrm{P} 11, \mathrm{P} 12 / \mathrm{P} 11, \mathrm{P} 33 / \mathrm{P} 11$, and $\mathrm{P} 34 / \mathrm{P} 11$. Other elements of the normalized scattering phase matrix are either zero or equal to these ones.

Figures 1 to 3 show the elements of the normalized scattering phase matrix of spherical perfectly conducting and sand particles in free space for size parameters $\left(2 \pi a / \lambda_{0}\right)$ of 10 , 30 , and 90, respectively. We can see that for different scattering angles the variations of $\mathrm{P} 11, \mathrm{P} 12 / \mathrm{P} 11, \mathrm{P} 33 / \mathrm{P} 11$, and P34/P11 for perfectly conducting particles and sand particles are very different. For metallic particles, when scattering angle is larger than $\sim 90^{\circ}, \mathrm{P} 11$, P12/P11, P33/P11, and P34/P11 change little with scattering angles. The larger the particle size parameter, the faster these elements approach to constant values. Particularly, when scattering angle is larger than $\sim 90^{\circ}$, the linear polarization degree of scattered light from metallic particles approaches to zero. However, the dielectric particles such as the sand spheres do not hold similar scattering properties, and have significant difference between horizontally and vertically polarized scattered lights at the backward scattering directions. Therefore with measuring the linear polarization degree of scattered light at backward scattering angles, metallic spheres can be unambiguously distinguished from dielectric ones. When there is an absorbing medium around the particle, as shown in Figs. 4 to 6, P11 has a trend of increase with the increase of scattering angle when scattering angle is larger than $\sim 90^{\circ}$ whereas other elements are still similar to those in free space.

Figures 7 to 9 show the elements of the normalized scattering phase matrix of perfectly conducting and sand circular cylinders in free space for size parameters $\left(2 \pi a / \lambda_{0}\right)$ of 10 , 30, and 90, respectively. Comparing Figs. 7 to 9 with Figs. 1 to 3, we can see that P11 for metallic circular cylinders significantly increase with the scattering angle when scattering 
angle is larger than $\sim 90^{\circ}$, but $\mathrm{P} 12 / \mathrm{P} 11, \mathrm{P} 33 / \mathrm{P} 11$, and $\mathrm{P} 34 / \mathrm{P} 11$ are still similar to those of spheres, changing little with scattering angles and approaching to constant values faster for larger particle size parameters. When the metallic circular cylinders are immersed in an absorbing host medium, as shown in Figs. 10 to 12, their elements of the normalized scattering phase matrix are similar to those in free space, especially the polarization ones. Because near constant values of the major elements except P11, of the normalized scattering phase matrix are such a general phenomena for various sized metallic particles in backward scattering directions, the integrated values for these elements over metallic aerosol size spectra should have the similar constants as calculated here at the scattering angles larger than $\sim 90^{\circ}$. Therefore, it is a doable task to detect and monitor metallic aerosol outbreak using classic polarimeters. Note that we only discussed the system illuminated by monochromatic light here. Although this is enough for narrowband detection or remote sensing applications, it is still important to consider the problem that the system is illuminated by polychromatic light. For light scattering by perfectly conducting particles, the effect of different wavelengths in a polychromatic light is simply the effect of different size parameters. From the results of different size parameters, we can see that the linear polarization of the scattered lights from metallic aerosols at scattering angles larger than $\sim 90^{\circ}$ is close to zero no matter what the particle size parameter is. Therefore, when the system is illuminated by polychromatic light we can still use linear polarization of the scattered lights at scattering angles larger than $\sim 90^{\circ}$ to identify metallic aerosols. 


\section{Conclusions}

In this work, light scattering characteristics of these metallic aerosols are studied using exact solutions on perfectly conducting spherical and cylindrical particles. It is found that for perfectly conducting spheres and cylinders, when scattering angle is larger than $\sim 90^{\circ}$ the linear polarization degree of the scattered light is very close to zero. This light scattering characteristics of perfectly conducting particles is significantly different from that of other aerosols. When these perfectly conducting particles are immersed in an absorbing medium, this light scattering characteristics does not show significant change. Therefore, measuring the linear polarization of scattered lights at backward scattering angles can detect and distinguish metallic particulates from other aerosols. This result provides a great potential of metallic aerosol detection and monitoring for environmental protection. 


\section{References}

1. Matli CS. Airborne metallic particulates from urban sources in a growing tropical town. International Association of Meteorology and Atmospheric Sciences, Beijing, China, August 2-11, 2005.

2. Bohren CF, Huffman DR. Absorption and scattering of light by small particles. Wiley, New York, 1983.

3. Mie G. Beigrade zur optik truber medien, speziell kolloidaler metallosungen. Ann Physic 1908; 25: 377-445.

4. Erma VA. An exact solution for the scattering of electromagnetic waves from conductors of arbitrary shape. I. Case of cylindrical symmetry. Phys Rev 1968; 173: $1243-57$.

5. Rayleigh. The dispersal of light by a dielectric cylinder. Phil Mag 1918; 36: 36576.

6. Padmabandu GG, Abromson D, Bickel WS. Light scattering by micron-sized conducting fibers: an experimental determination. Appl Opt 1991; 30: 139-44.

7. Purcell EM, Pennypacker CP. Scattering and absorption of light by nonspherical dielectric grains. Astrophys J 1973; 196: 705-14.

8. Singham SB, Bohren CF. Light scattering by an arbitrary particle: a physical reformation of the coupled dipole method. Opt Lett 1987; 12: 10-2.

9. Draine BT. The discrete-dipole approximation and its application to interstellar graphite grains. Astrophys J 1988; 333: 848-72.

10. Yee KS. Numerical solution of initial boundary value problems involving Maxwell's equation in isotropic media. IEEE Trans Antennas Propag 1966; AP14: 302-7. 
11. Yang P, Liou KN. Finite-difference time domain method for light scattering by small ice crystals in three-dimensional space. J Opt Soc Am A 1996; 13: 2072-85.

12. Sun WB, Fu Q, Chen ZZ. Finite-difference time-domain solution of light scattering by dielectric particles with a perfectly matched layer absorbing boundary condition. Appl Opt 1999; 38: 3141-51.

13. Sun WB, Loeb NG, Fu Q. Finite-difference time-domain solution of light scattering and absorption by particles in an absorbing medium. Appl Opt 2002; 41: 5728-43.

14. Li X, Taflove A, Backman V. Modified FDTD near-to-far-field transformation for improved backscattering calculation of strongly forward-scattering objects. IEEE Antennas Wireless Propag Lett 2005; 4: 35-8.

15. Fu Q, Sun WB. Mie theory for light scattering by a spherical particle in an absorbing medium. Appl Opt 2001; 40: 1354-61.

16. Sun WB, Loeb NG, Lin B. Light scattering by an infinite circular cylinder immersed in an absorbing medium. Appl Opt 2005; 44: 2338-42. 


\section{Figure Captions}

Fig. 1. The elements of the normalized scattering phase matrix of perfectly conducting and sand spheres with a size parameter $\left(2 \pi a / \lambda_{0}\right)$ of 10 in free space. A refractive index of 1.5 is used for sand.

Fig. 2. Same as Fig. 1, but the size parameter is 30.

Fig. 3. Same as Fig. 1, but the size parameter is 90.

Fig. 4. The elements of the normalized scattering phase matrix of perfectly conducting and sand spheres with a size parameter $\left(2 \pi a / \lambda_{0}\right)$ of 10 in an absorbing medium with a refractive index of $1.33+0.01$ i. A refractive index of 1.5 is used for sand.

Fig. 5. Same as Fig. 4, but the size parameter is 30.

Fig. 6. Same as Fig. 4, but the size parameter is 90.

Fig. 7. The elements of the normalized scattering phase matrix of perfectly conducting and sand circular cylinders with a size parameter $\left(2 \pi a / \lambda_{0}\right)$ of 10 in free space. A refractive index of 1.5 is used for sand.

Fig. 8. Same as Fig. 7, but the size parameter is 30 . 
Fig. 9. Same as Fig. 7, but the size parameter is 90.

Fig. 10. The elements of the normalized scattering phase matrix of perfectly conducting and sand circular cylinders with a size parameter $\left(2 \pi a / \lambda_{0}\right)$ of 10 in an absorbing medium with a refractive index of $1.33+0.01$ i. A refractive index of 1.5 is used for sand.

Fig. 11. Same as Fig. 10, but the size parameter is 30.

Fig. 12. Same as Fig. 10, but the size parameter is 90. 

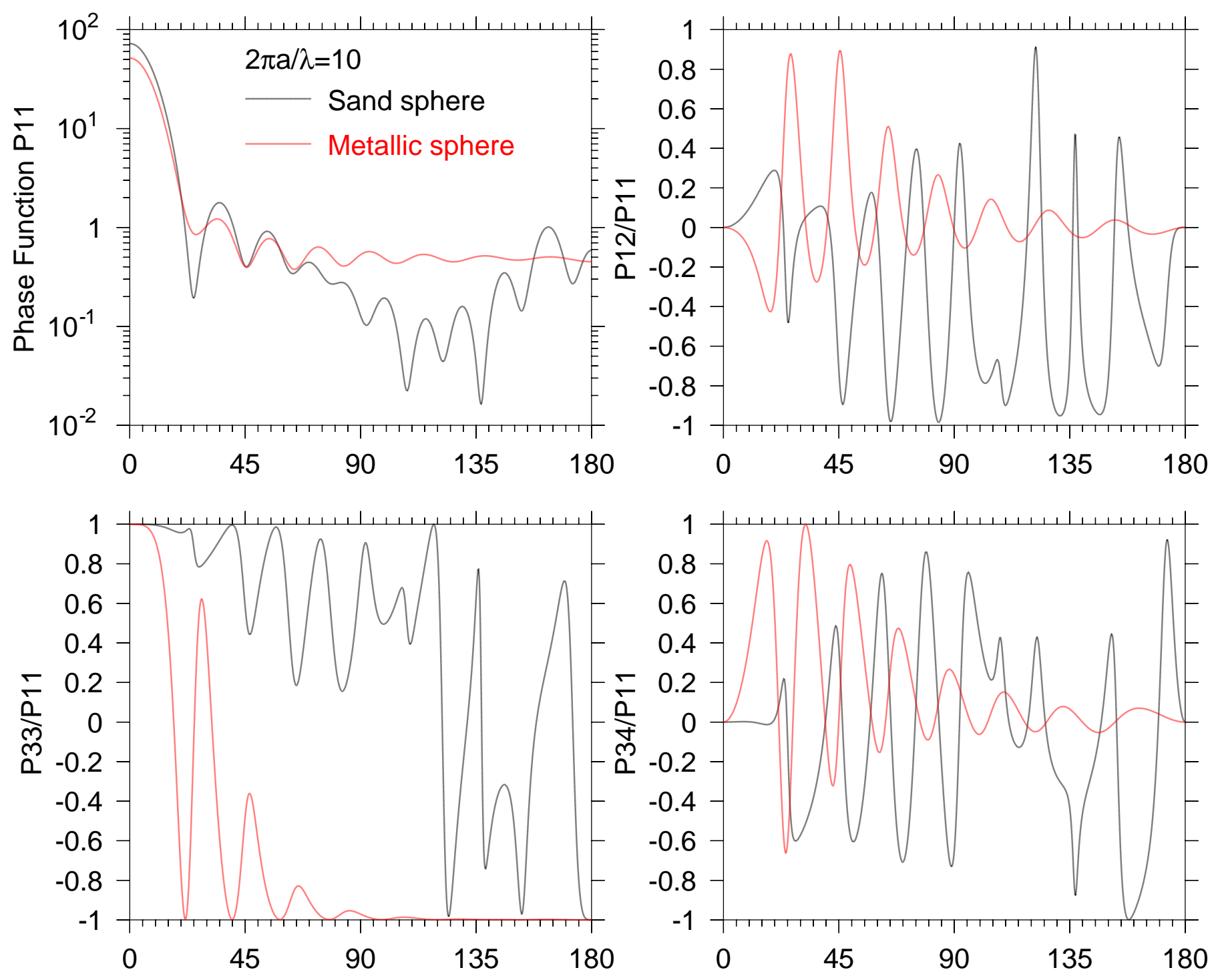

Figure 1 of Sun 

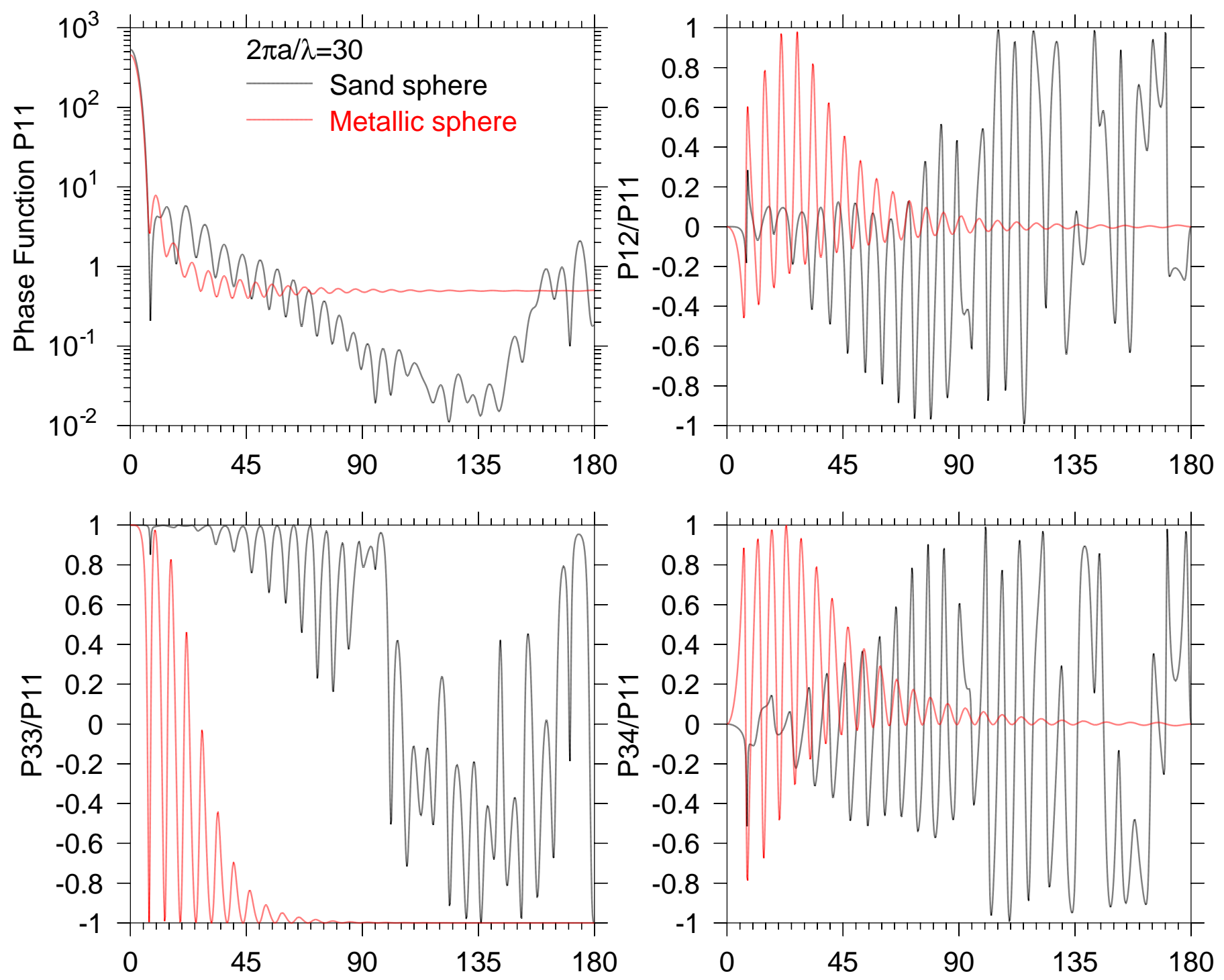

Figure 2 of Sun 

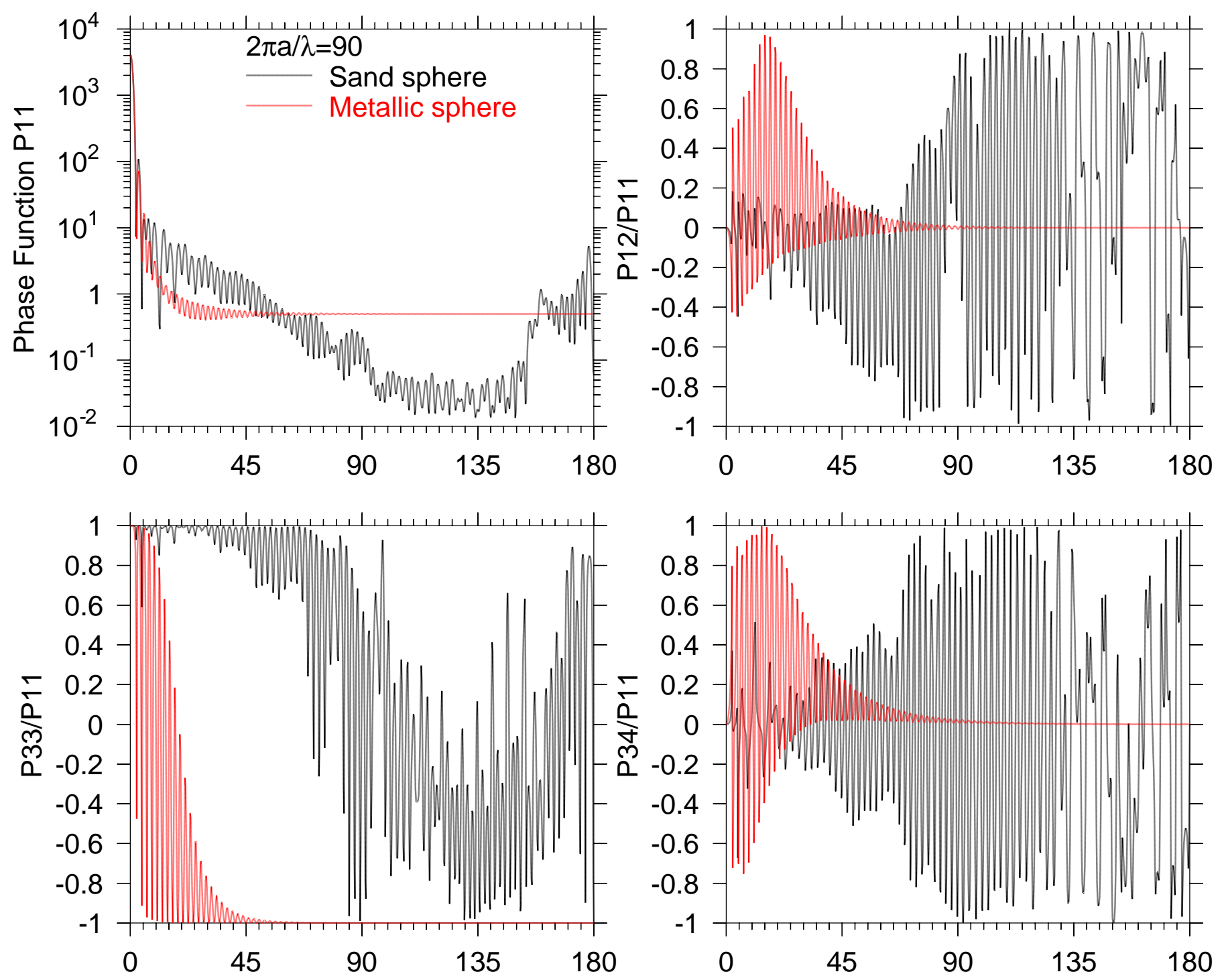

Figure 3 of Sun 

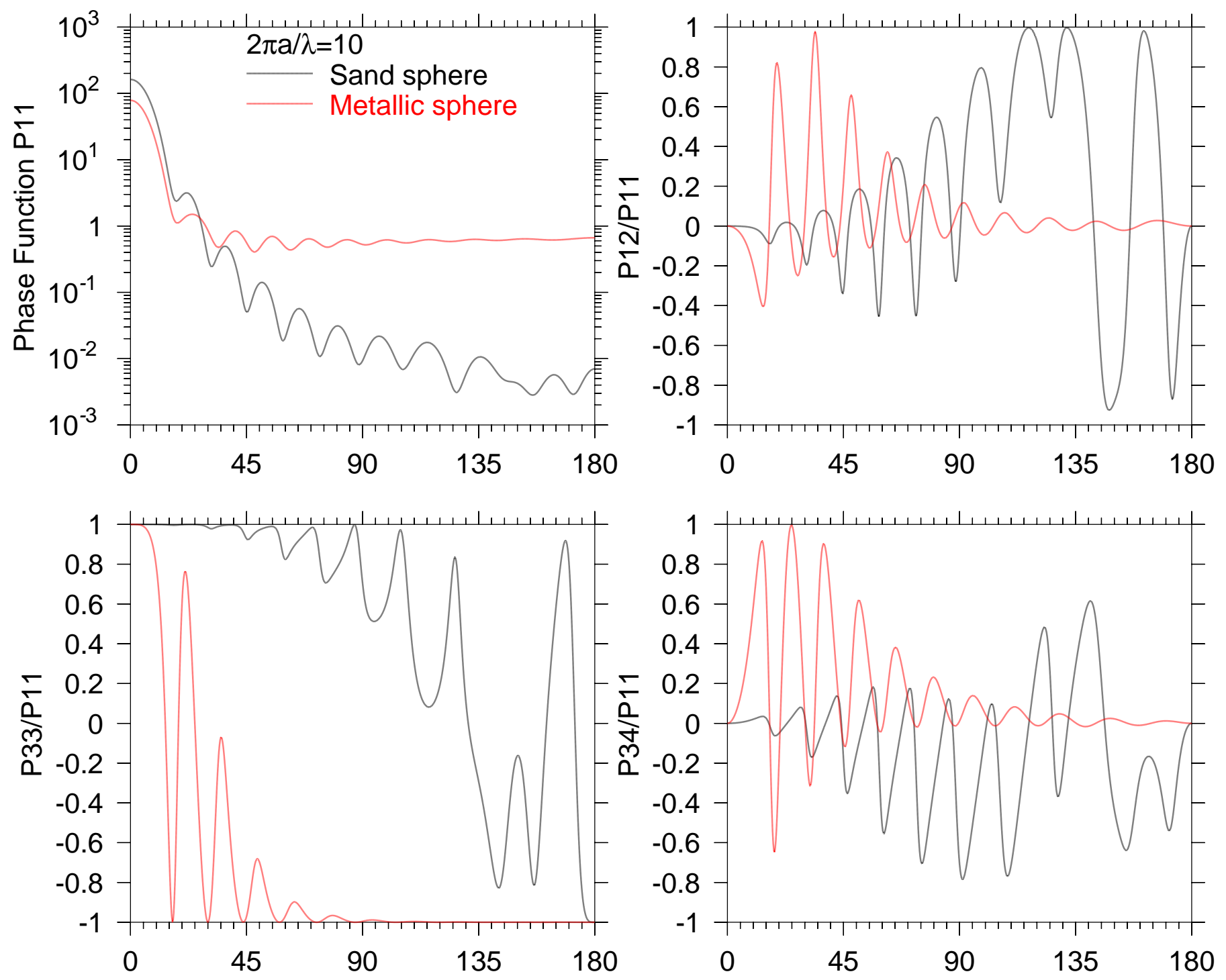

Figure 4 of Sun 

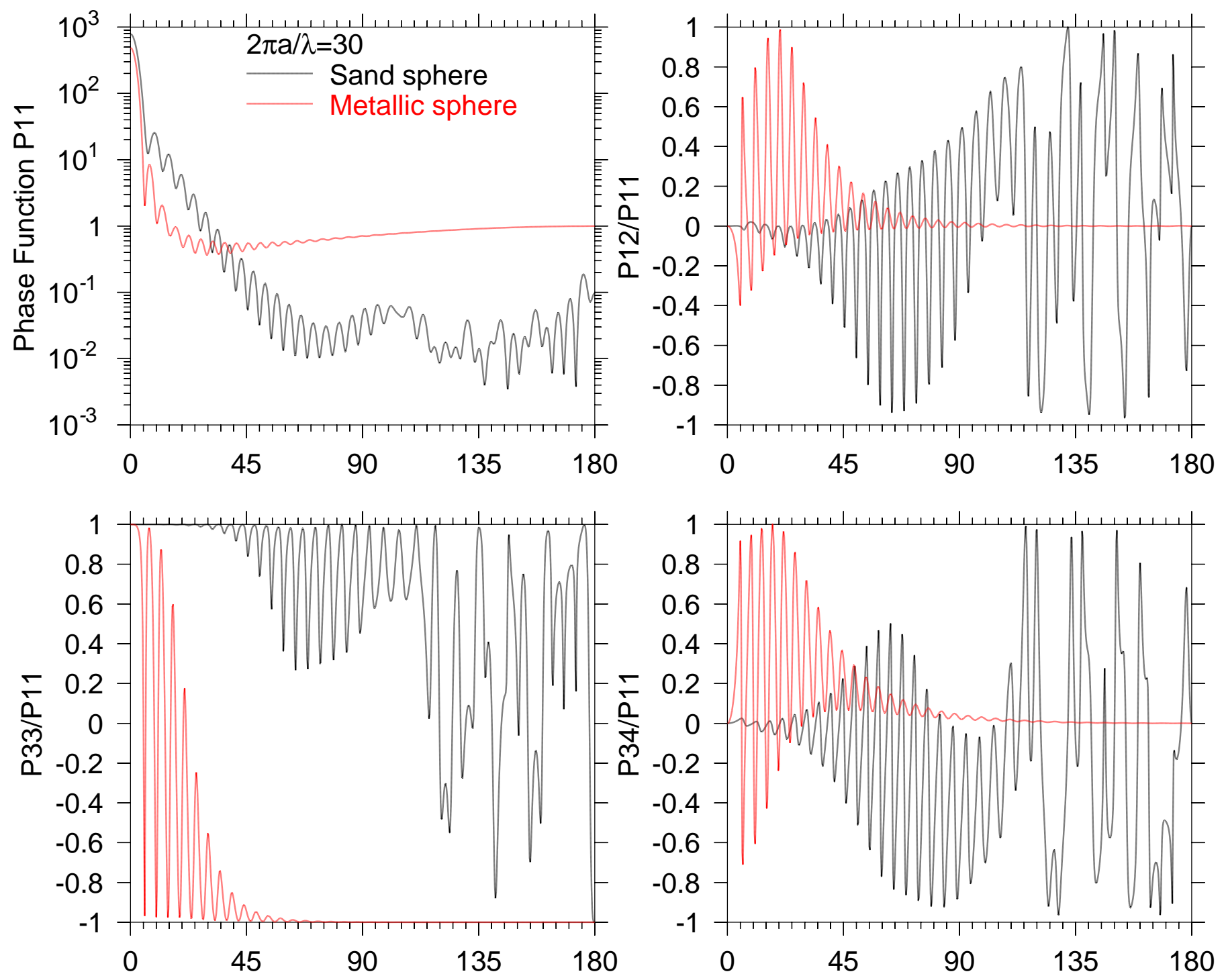

Figure 5 of Sun 

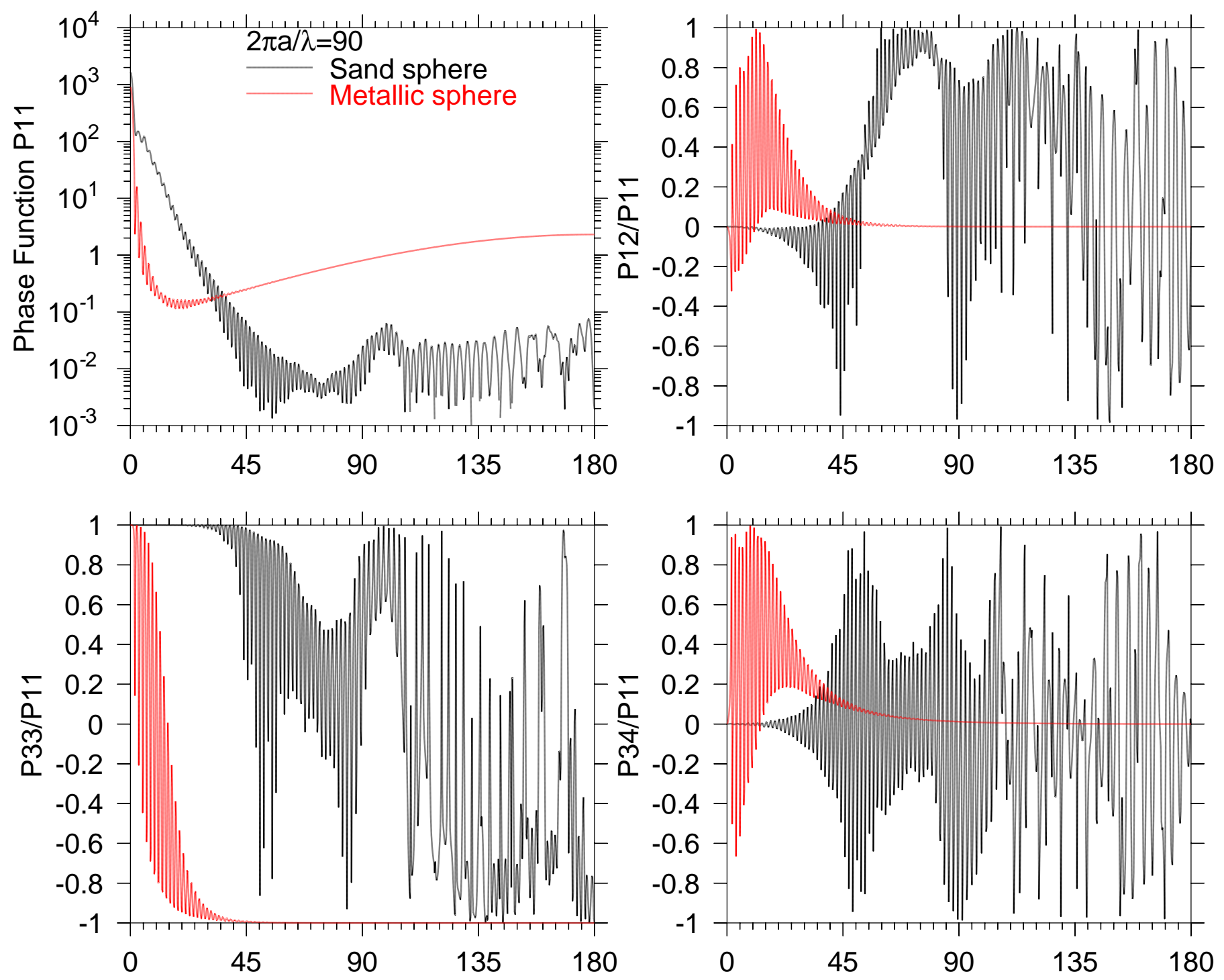

Figure 6 of Sun 

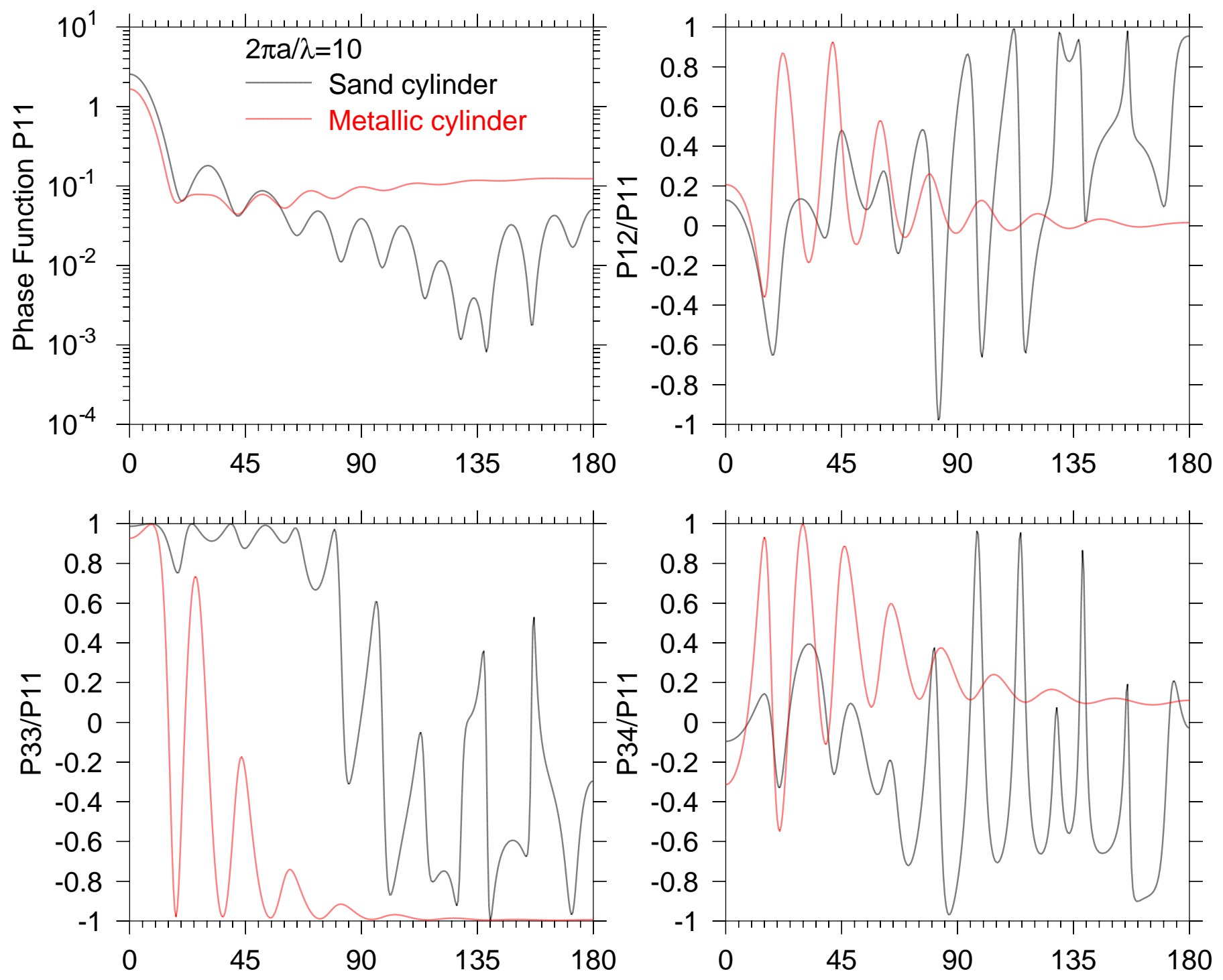

Figure 7 of Sun 

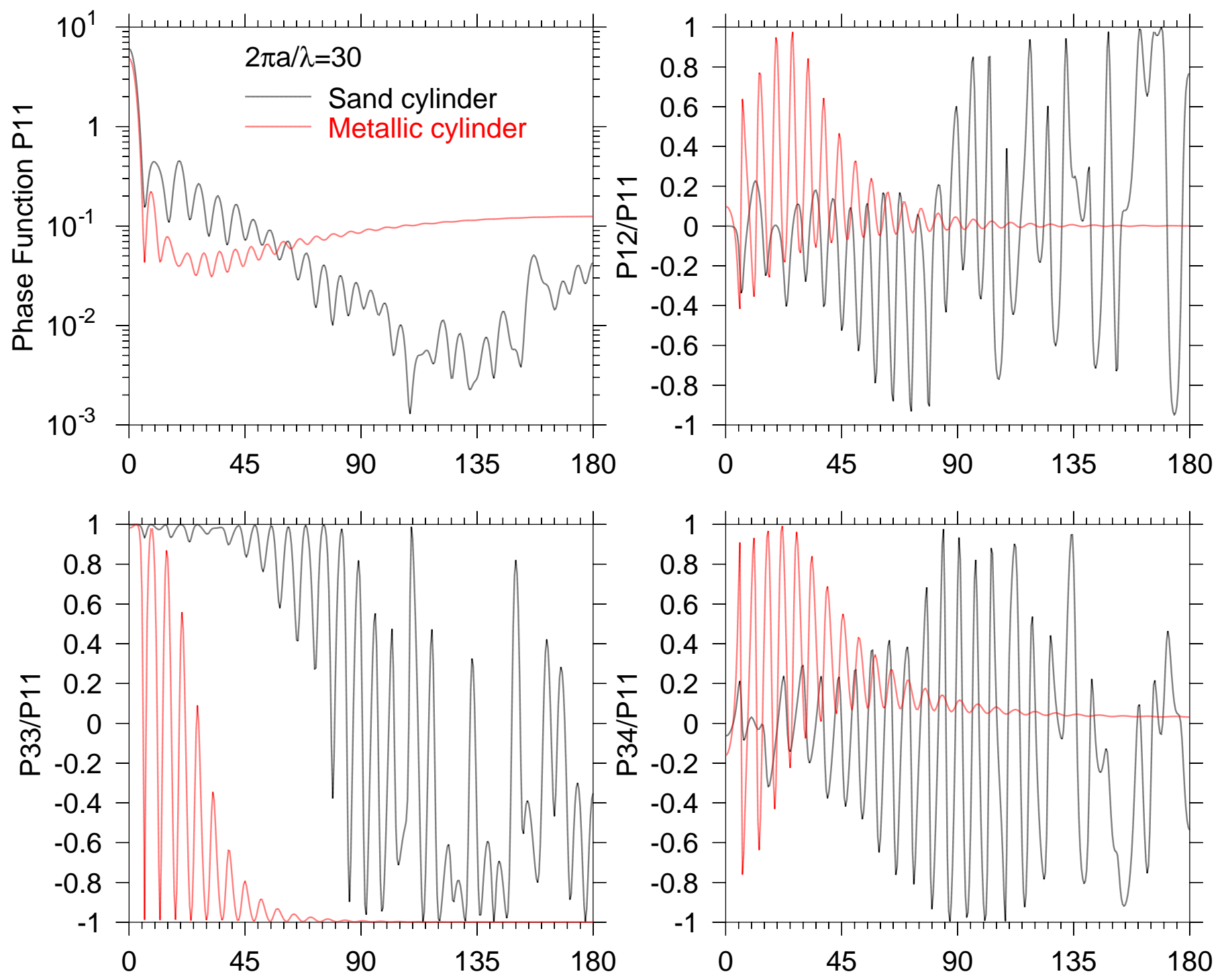

Figure 8 of Sun 

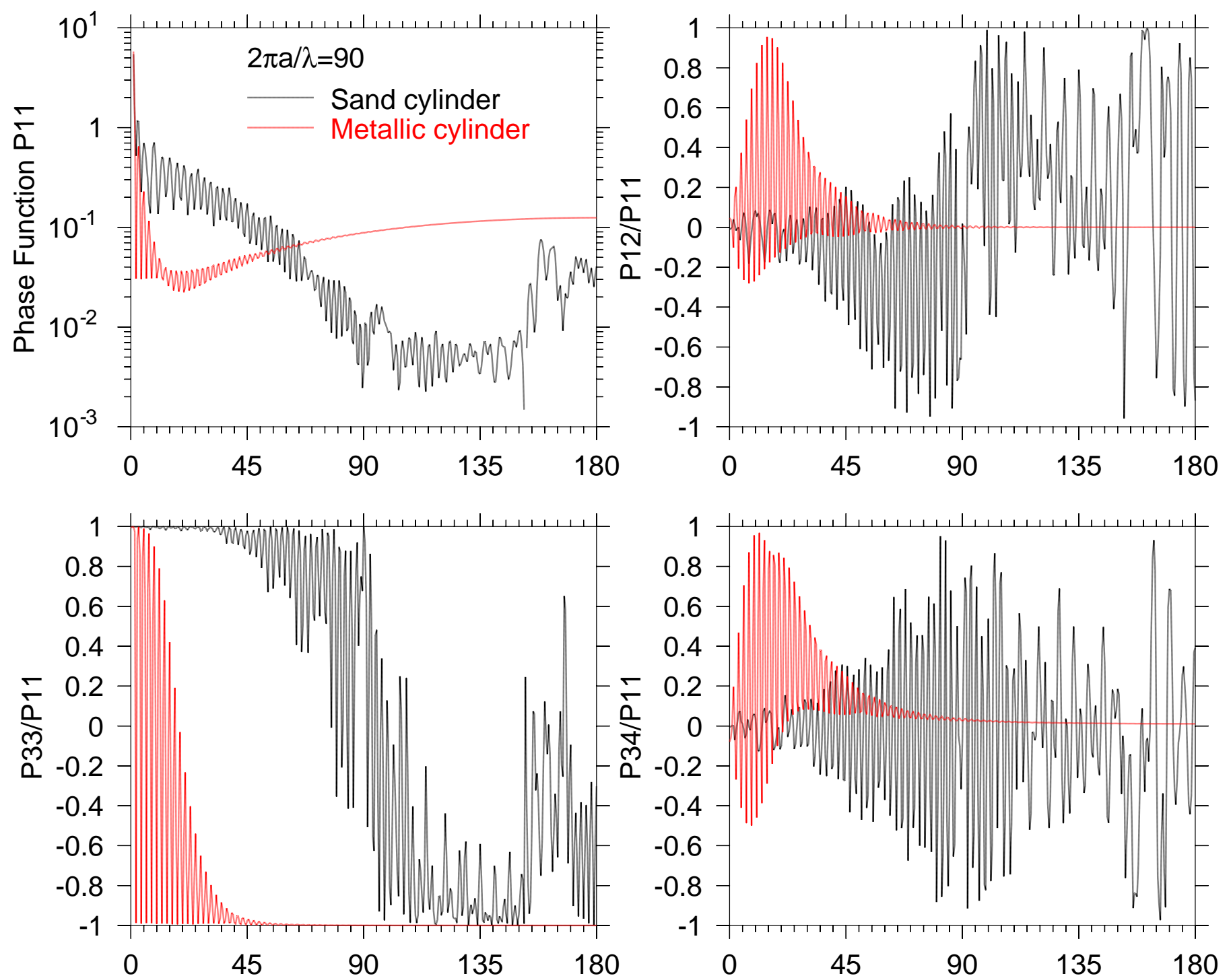

Figure 9 of Sun 

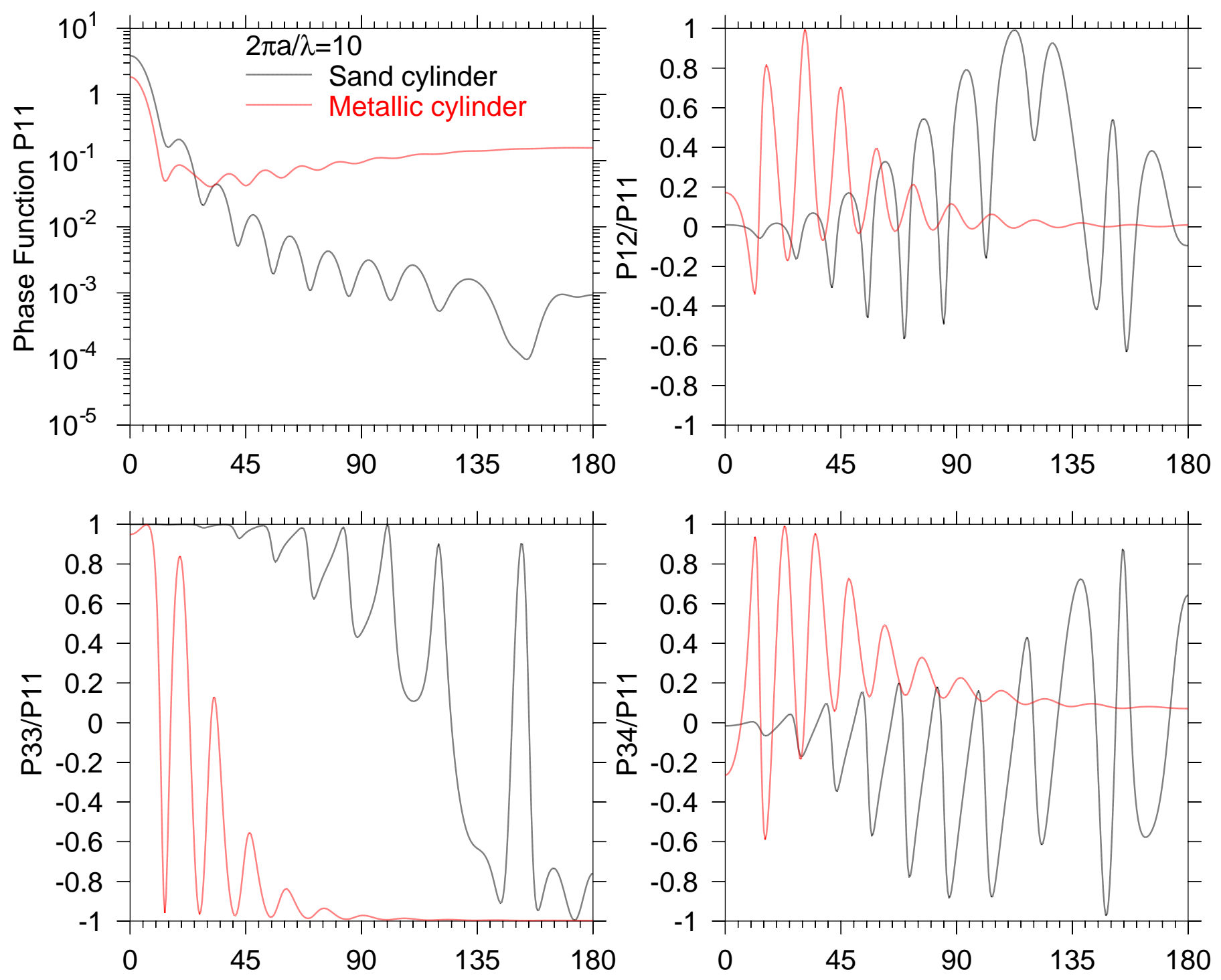

Figure 10 of Sun 

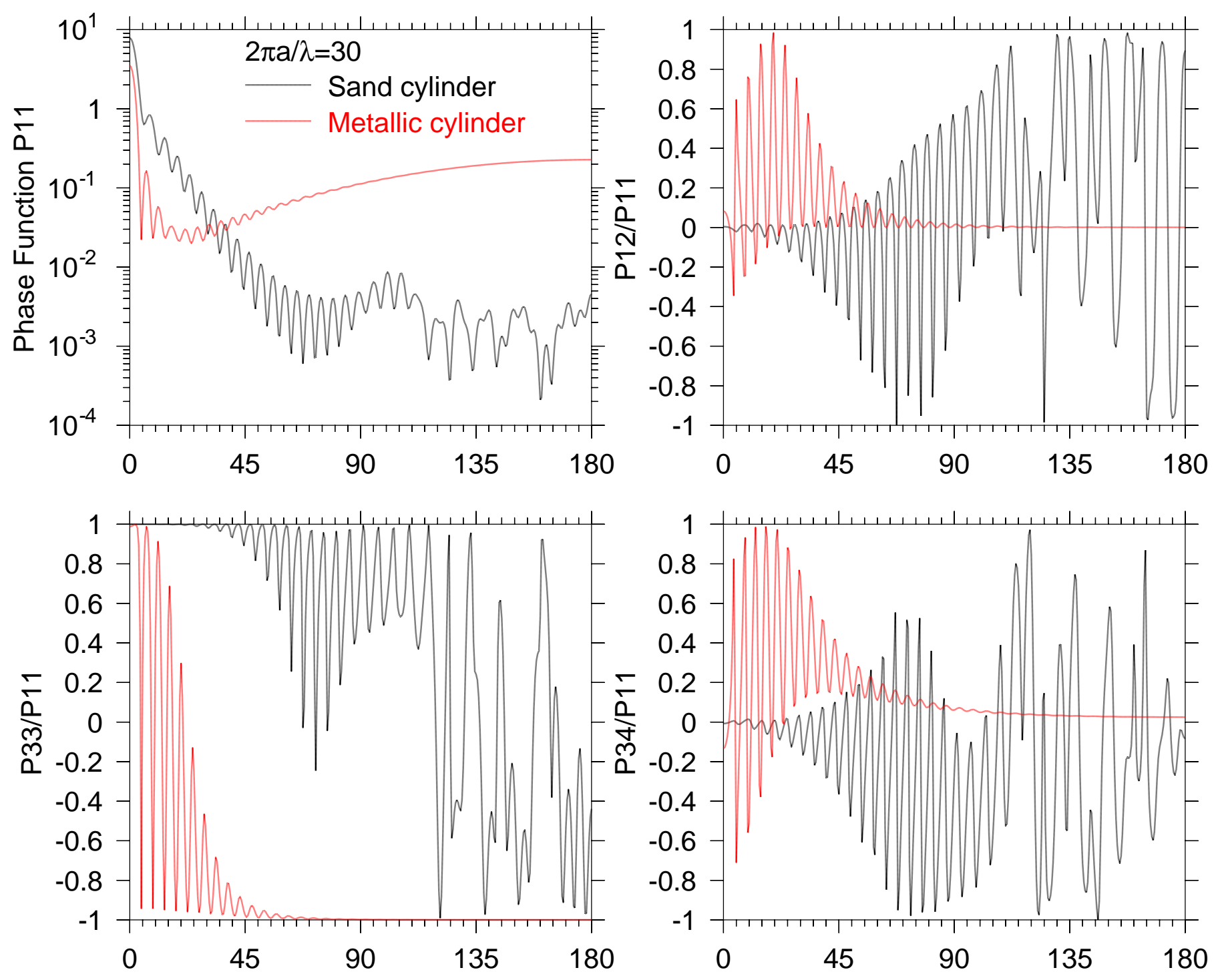

Figure 11 of Sun 

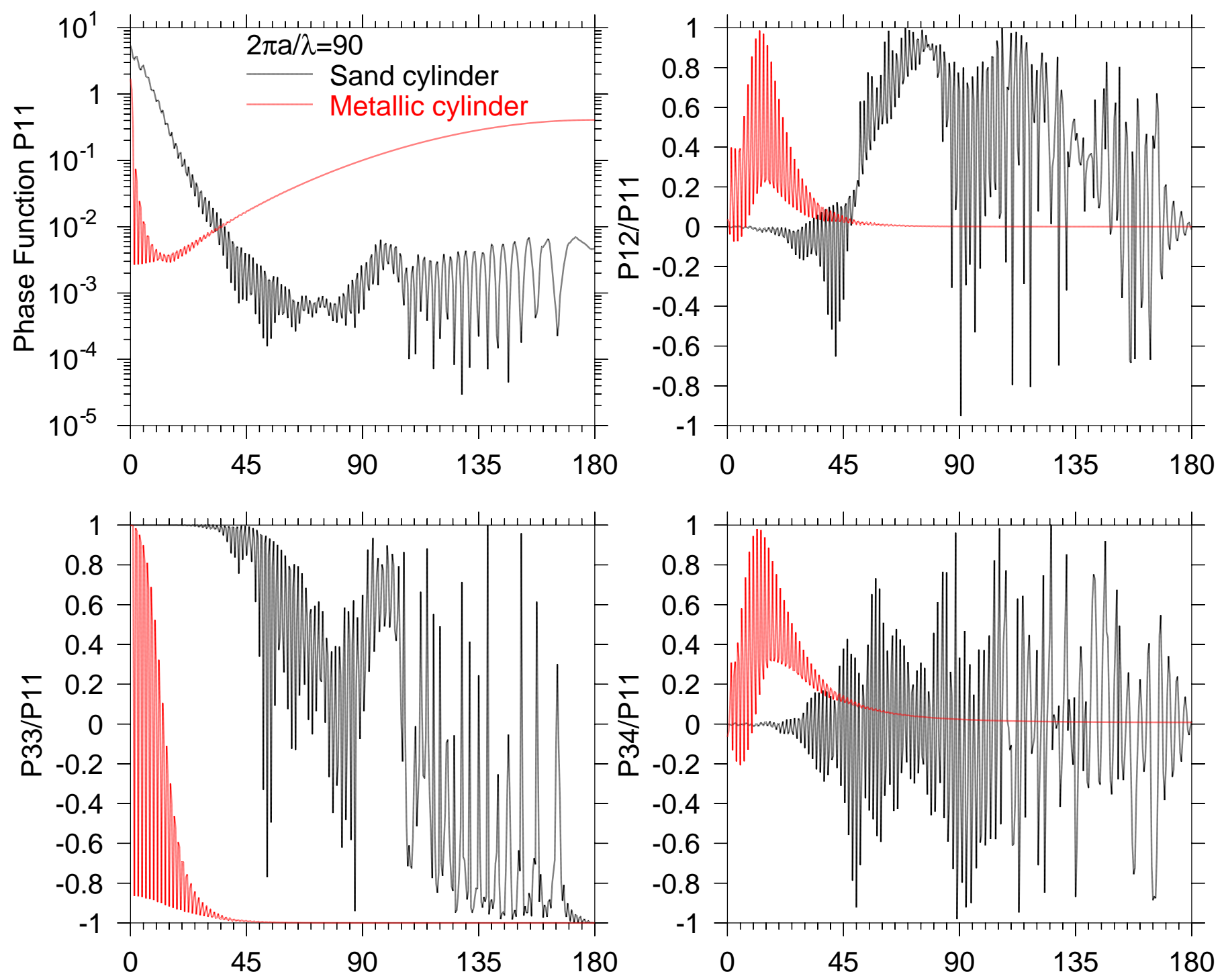

Figure 12 of Sun 